\title{
A!
}

This is an electronic reprint of the original article.

This reprint may differ from the original in pagination and typographic detail.

Pihko, Ainoliisa J.; Nicolaou, K.C.; Koskinen, Ari M.P.

\section{An expedient synthesis of D-callipeltose}

\section{Published in:}

TETRAHEDRON-ASYMMETRY

DOI:

10.1016/S0957-4166(01)00146-X

Published: 01/01/2001

Document Version

Peer reviewed version

Please cite the original version:

Pihko, A. J., Nicolaou, K. C., \& Koskinen, A. M. P. (2001). An expedient synthesis of D-callipeltose.

TETRAHEDRON-ASYMMETRY, 12, 937-942. https://doi.org/10.1016/S0957-4166(01)00146-X

This material is protected by copyright and other intellectual property rights, and duplication or sale of all or part of any of the repository collections is not permitted, except that material may be duplicated by you for your research use or educational purposes in electronic or print form. You must obtain permission for any other use. Electronic or print copies may not be offered, whether for sale or otherwise to anyone who is not an authorised user. 


\title{
An expedient synthesis of D-callipeltose
}

\author{
Ainoliisa J. Pihko, ${ }^{\mathrm{a}, \mathrm{b}}$ K. C. Nicolaou ${ }^{\mathrm{a}, *}$ and Ari M. P. Koskinen ${ }^{\mathrm{b}, *}$ \\ ${ }^{a}$ Department of Chemistry and The Skaggs Institute for Chemical Biology, The Scripps Research Institute, \\ 10550 North Torrey Pines Road, La Jolla, CA 92037, USA and Department of Chemistry and Biochemistry, \\ University of California, San Diego, 9500 Gilman Drive, La Jolla, CA 92093, USA \\ ${ }^{\mathrm{b}}$ Laboratory of Organic Chemistry, Department of Chemical Technology, Helsinki University of Technology, \\ FIN-02015 HUT, Finland
}

\begin{abstract}
Methyl D-callipeltose $\mathbf{1 2}$ and D-callipeltose $\mathbf{4}$ were synthesized from D-glucal $\mathbf{5}$ in 10 and 11 steps, respectively. The synthesis features an azide displacement reaction of an $\alpha$-nosyloxy ketone 7 and a highly diastereoselective $C$-methylation of $\alpha$-azido ketone 8. (C) 2001 Elsevier Science Ltd. All rights reserved.
\end{abstract}

\section{Introduction}

Callipeltoside A 1 belongs to a new class of cyclodepsipeptides called callipeltins. The molecules were isolated in 1996 from the shallow water lithistid sponge Callipelta sp., a native of New Caledonia..$^{1,2}$ They were found to inhibit in vitro proliferation of $\mathrm{KB}$ and $\mathrm{P} 338$ cells and to protect cells infected with HIV. Structurally, 1 consists of a macrocyclic lactone linked to a unique dienyne cyclopropane side chain and the deoxy amino sugar, callipeltose, 4 (Fig. 1). The absolute stereochemistry of the molecule has not been established.

There has been considerable synthetic interest in $\mathbf{1}$ although no total synthesis has been reported to date. ${ }^{3-7}$
The deoxy amino sugar 4 contains an unusual fivemembered oxazolidinone ring fused to the sugar backbone at positions 3 and 4 . Both enantiomers of 4 have been synthesized-methyl L-callipeltose in 10 steps from L-rhamnose ${ }^{8}$ and methyl D-callipeltose in 14 steps from methyl D-mannose. ${ }^{9}$ Herein we report an expedient synthesis of $\alpha$-D-callipeltose $\mathbf{4}$ from D-glucal 5 .

\section{Results and discussion}

The $\alpha, \beta$-unsaturated ketone $\mathbf{6}$ was synthesized from D-glucal in three steps (Scheme 1). ${ }^{10} \mathrm{~A}$ sequence of tosylation (TsCl, pyr.) ${ }^{11}$ and reduction with $\mathrm{LiAlH}_{4}{ }^{12,13}$ effected deoxygenation of the 6-position of D-glucal 5 .

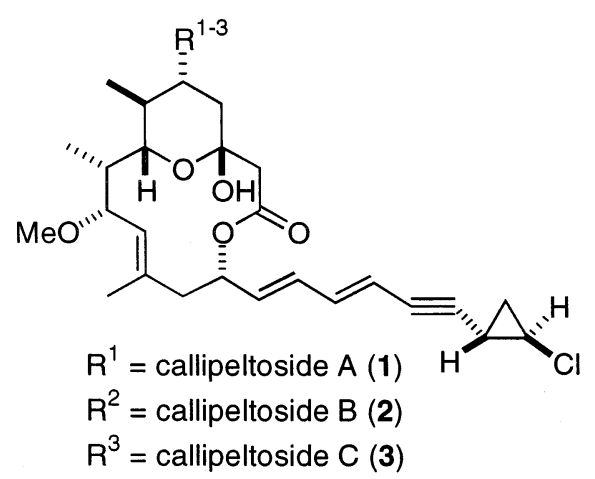

Figure 1. Structures of callipeltosides A-C, 1-3.<smiles>[Y]C1OC(C)C2NC(=O)O[C@@]2(C)C1OC</smiles><smiles>COC1C(O)OC(C)C(N=C=O)[C@@]1(C)O</smiles><smiles>COC1C(O)OC(C)[C@H](O)[C@@]1(C)O</smiles>

$\mathrm{X}=\mathrm{O}: \mathrm{R}^{1}$

$\mathrm{X}=\mathrm{OH}$ : D-callipeltose $(\mathbf{4})$ 

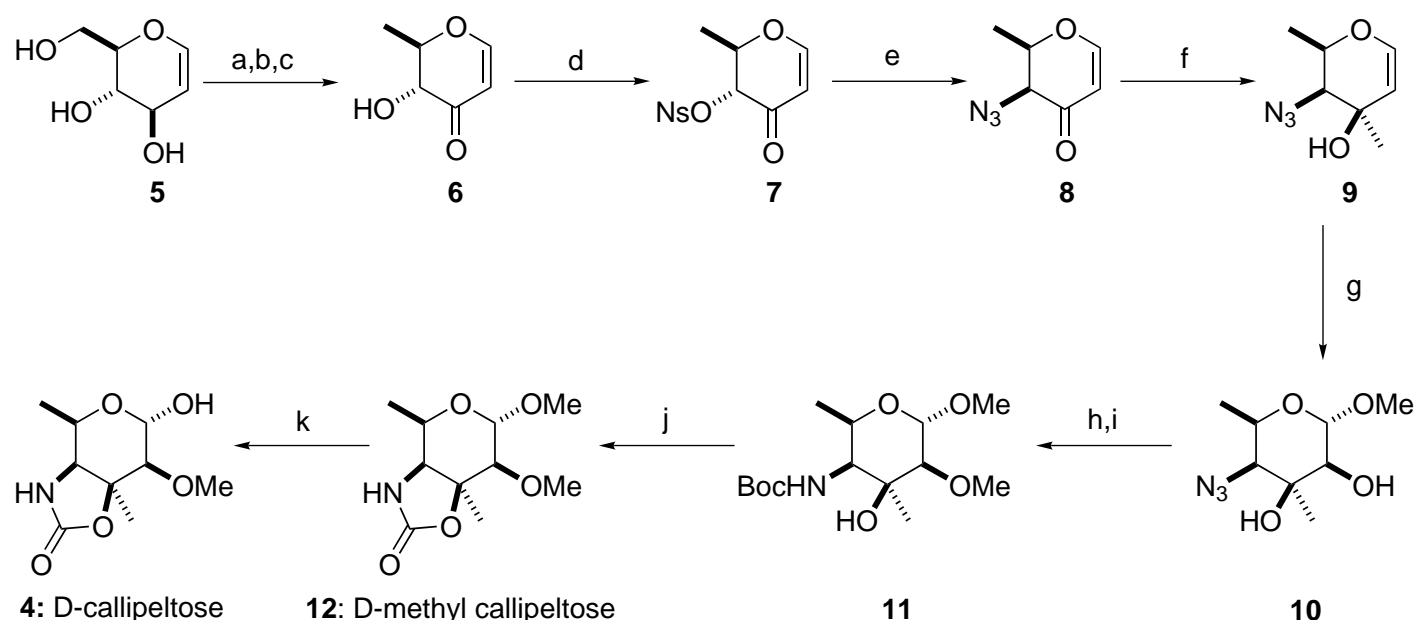

Scheme 1. Synthesis of D-callipeltose. Reagents and conditions: (a) TsCl, py, $\mathrm{CH}_{2} \mathrm{Cl}_{2}, 0^{\circ} \mathrm{C} \rightarrow \mathrm{rt}, 78 \%$; (b) $\mathrm{LiAlH}$, $\mathrm{THF}, \Delta, 59 \%$; (c) $\mathrm{MnO}_{2}, \mathrm{CH}_{2} \mathrm{Cl}_{2}, \mathrm{rt}, 66 \%$; (d) $\mathrm{NsCl}, \mathrm{py}, \mathrm{CH}_{2} \mathrm{Cl}_{2}, 0^{\circ} \mathrm{C} \rightarrow \mathrm{rt}, 80 \%$; (e) $n-\mathrm{Bu}_{4} \mathrm{NN}_{3}, \mathrm{CH}_{2} \mathrm{Cl}_{2}, 0^{\circ} \mathrm{C} \rightarrow \mathrm{rt}, 95 \%$; (f) $\mathrm{MeLi}, \mathrm{THF},-100^{\circ} \mathrm{C}$, $77 \%$; (g) $m$-CPBA, $\mathrm{NaHCO}_{3}, \mathrm{MeOH}, 0^{\circ} \mathrm{C} \rightarrow \mathrm{rt}, 60 \%$; (h) $\mathrm{MeI}, \mathrm{Ag}_{2} \mathrm{O}, \mathrm{Et}_{2} \mathrm{O}, \Delta, 72 \%$; (i) $\mathrm{H}_{2}, 10 \% \mathrm{Pd} / \mathrm{C}, \mathrm{Boc} 2 \mathrm{O}, \mathrm{EtOAc}, \mathrm{rt}, 62 \%$; (j) $t$-BuOK, THF, $0^{\circ} \mathrm{C} \rightarrow \mathrm{rt}, 69 \%$; (k) $2 \mathrm{M} \mathrm{H}_{2} \mathrm{SO}_{4}$ in $1: 1 \mathrm{H}_{2} \mathrm{O} /$ dioxane, $60-70^{\circ} \mathrm{C}, 48 \%$. $\mathrm{Rt}=$ room temperature, $\mathrm{NsCl}=\mathrm{nosyl}$ chloride, $\mathrm{py}=$ pyridine, $m-\mathrm{CPBA}=m$-chloroperbenzoic acid, $\mathrm{Boc}=t$-butoxycarbonyl.

The resulting D-rhamnal was oxidized with $\mathrm{MnO}_{2}{ }^{14}$ to give $\alpha, \beta$-unsaturated ketone $\mathbf{6}$. The next task was to introduce a nitrogen atom at the 4-position of the sugar by azide displacement of a suitable leaving group.

For this purpose, several leaving groups, including mesylate, ${ }^{15}$ triflate $^{15}$ and imidazolyl sulfonate, ${ }^{16}$ were examined, but only the nosylate group ${ }^{17}$ could be displaced at an acceptable rate without decomposition. The nosylate 7 was obtained by the action of nosyl chloride and pyridine on $\mathbf{6}$ in $80 \%$ yield along with ca. $3-5 \%$ of its C-(4) epimer. Sodium azide was found to be too basic for the displacement reaction; stirring 7 overnight in DMSO with $\mathrm{NaN}_{3}$ at room temperature gave, in addition to recovered starting material, ca. 1:1 mixture of both epimers of $\mathbf{8}$, presumably due to product enolization. However, tetra- $n$-butylammonium azide $^{18,19}$ furnished the desired azide 8 in $90 \%$ yield along with $5-10 \%$ of its C-(4) epimer. ${ }^{20}$ The $C$-methylation with methyllithium in THF at $-100^{\circ} \mathrm{C}$ gave the tertiary alcohol 9 in $77 \%$ yield as a single diastereomer. ${ }^{14,21}$ The observed selectivity is rationalized through a synergy of steric and stereoelectronic considerations, as described in Fig. 2: the axially disposed azide group prevents attack from the $R e$ face of the carbonyl, and the electronegativity of the nitrogen favors attack from the opposite $(\mathrm{Si})$ face.

The next objective was to install the final two stereocenters of the sugar. Epoxidation accompanied by concomitant stereo- and regioselective opening of the epoxide is a useful method to functionalize the double bond in glucals. ${ }^{22-28}$ Thus, reaction of 9 with $m$-CPBA in methanol in the presence of $\mathrm{NaHCO}_{3}$ led to the stereoselective formation of $\mathbf{1 0}$ in $60 \%$ yield. Methylation of the newly created alcohol with $\mathrm{MeI}$ and $\mathrm{Ag}_{2} \mathrm{O}^{29}$ cleanly alkylated the secondary hydroxyl group. Subsequent palladium-catalyzed hydrogenation reduced the azide to the corresponding amine, which, in the pres-

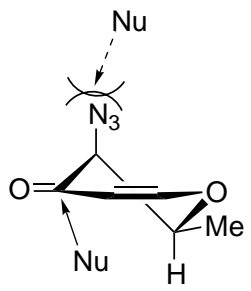

Figure 2. Preferred approach of nucleophile in the conversion of 8 to 9 .

ence of Boc-anhydride, furnished amide $\mathbf{1 1},{ }^{30}$ setting the stage for the oxazolidinone ring closure. Thus, exposure of the tertiary alcohol $\mathbf{1 1}$ to potassium $t$-butoxide in THF, following the procedure of Davies et al., furnished the methyl glycoside 12. ${ }^{31}$ Finally, the glycoside was hydrolyzed with $2 \mathrm{M}$ sulfuric acid in water/dioxane $(1: 1)$ at $60^{\circ} \mathrm{C},{ }^{32,33}$ concluding the synthesis of D-callipeltose 4 in 11 steps from commercially available D-glucal 5.

\section{Conclusion}

In conclusion, methyl D-callipeltose $\mathbf{1 2}$ and D-callipeltose 4 were synthesized in a highly selective manner starting from D-glucal 5 in 10 and 11 steps, respectively. Key features of this synthesis, the shortest reported to date for 4, include: (i) readily available starting materials; (ii) use of an $\alpha$-nosyloxy ketone 7 en route to azide 8; and (iii) a highly diastereoselective $C$-methylation rationalized in Fig. 2.

\section{Experimental}

All reactions were carried out under an argon atmosphere with dry solvents under anhydrous conditions, if 
necessary. Anhydrous solvents were obtained by passing them through commercially available activated alumina columns. Yields refer to chromatographically and spectroscopically ( ${ }^{1} \mathrm{H}$ NMR) homogeneous materials, unless otherwise stated. All reagents were purchased at highest commercial quality and used without further purification, unless otherwise stated. All reactions were monitored by thin-layer chromatography carried out on $0.25 \mathrm{~mm}$ E. Merck silica gel plates (60F-254) using UV light as visualizing agent and acidic PMA $(2.5 \%$ phosphomolybdic acid, $1.5 \% o$ phosphoric acid and 5\% sulfuric acid in water) or $1 \%$ aqueous potassium permanganate solution and heat as developing agents. E. Merck silica gel (60, particle size $0.040-0.063 \mathrm{~mm}$ ) was used for flash column chromatography. NMR spectra were recorded on Bruker DRX-600, DRX-500 or AMX-400 instruments and calibrated using residual undeuterated solvents as an internal reference $\left(\mathrm{CDCl}_{3} 7.26 \mathrm{ppm}, \mathrm{MeOH} 3.34\right.$ $\mathrm{ppm}$ ). The following abbreviations are used to explain the multiplicities: $\mathrm{s}=$ singlet; $\mathrm{d}=$ doublet; $\mathrm{t}=$ triplet; $\mathrm{q}=$ quartet; $\mathrm{m}=$ multiplet; $\mathrm{b}=$ broad. IR spectra were recorded on a Perkin-Elmer 1600 series FT-IR spectrometer. Optical rotations were recorded on a PerkinElmer 241 polarimeter. High resolution mass spectra (HRMS) were recorded on an IonSpec mass spectrometer under MALDI-FTMS conditions with NBA or DHB as the matrix. Low resolution mass spectra were recorded on a Hewlett Packard 5971A benchtop GC/ MS. Melting points $(\mathrm{mp})$ are uncorrected and were recorded on a Thomas-Hoover Unimelt capillary melting point apparatus.

\subsection{D-Rhamnal}

To a stirred solution of D-glucal $5(2.50 \mathrm{~g}, 17.1 \mathrm{mmol})$ in pyridine $(42 \mathrm{~mL})$ and $\mathrm{CH}_{2} \mathrm{Cl}_{2}(42 \mathrm{~mL})$, cooled at $0^{\circ} \mathrm{C}$, was added tosyl chloride $(4.89 \mathrm{~g}, 25.7 \mathrm{mmol})$ and the cooling bath was removed. After stirring for $2.5 \mathrm{~h}$ at ambient temperature, the reaction mixture was cooled again to $0^{\circ} \mathrm{C}$, quenched with water $(5 \mathrm{~mL})$ and stirred for $30 \mathrm{~min}$. More water $(20 \mathrm{~mL})$ was added, the organic layer was separated and washed with sat. aq. $\mathrm{CuSO}_{4}(3 \times 20 \mathrm{~mL})$ and water $(3 \times 20 \mathrm{~mL})$. The combined aqueous phases (excluding the first water layer) were extracted with $\mathrm{CH}_{2} \mathrm{Cl}_{2}(2 \times 20 \mathrm{~mL})$ and those organic extracts were washed with water $(2 \times 10$ $\mathrm{mL}$ ). The combined organic extracts were washed with brine $(20 \mathrm{~mL})$, dried $\left(\mathrm{Na}_{2} \mathrm{SO}_{4}\right)$, filtered and concentrated to provide crude $6-O$-tosyl-D-glucal as a yellow oil (3.99 g, 78\% yield). $R_{\mathrm{f}} 0.55$ (EtOAc); ${ }^{1} \mathrm{H}$ NMR $\left(400 \mathrm{MHz}, \mathrm{CDCl}_{3}\right) \delta 7.81(\mathrm{~d}, J=8.2 \mathrm{~Hz}, 2 \mathrm{H}), 7.36(\mathrm{~d}$, $J=8.2 \mathrm{~Hz}, 2 \mathrm{H}), 6.24(\mathrm{dd}, J=6.1,1.6 \mathrm{~Hz}, 1 \mathrm{H}), 4.74$ $(\mathrm{dd}, J=6.1,2.1 \mathrm{~Hz}, 1 \mathrm{H}), 4.48(\mathrm{dd}, J=11.4,3.8 \mathrm{~Hz}$, 1H), 4.27 (app. d, $J=11.4 \mathrm{~Hz}, 2 \mathrm{H}), 3.91$ (ddd, $J=$ $10.0,3.8,2.1 \mathrm{~Hz}, 1 \mathrm{H}), 3.77(\mathrm{dd}, J=10.0,7.3 \mathrm{~Hz}, 1 \mathrm{H})$, $2.45(\mathrm{~s}, 3 \mathrm{H})$.

To a stirred solution of 6- $O$-tosyl-D-glucal (3.99 g, $13.3 \mathrm{mmol})$ in THF $(30 \mathrm{~mL})$ at $0^{\circ} \mathrm{C}$ was added $\mathrm{LiAlH}_{4}$ (0.9 $\mathrm{M}$ solution in THF, $44.2 \mathrm{~mL}, 39.9 \mathrm{mmol})$ dropwise and the reaction mixture was heated to reflux.
After stirring the reaction for $1 \mathrm{~h}$, the mixture was cooled to $0^{\circ} \mathrm{C}$ and quenched slowly with $\mathrm{H}_{2} \mathrm{O}(1.5$ $\mathrm{mL}), 15 \% \mathrm{NaOH}(1.5 \mathrm{~mL})$ and $\mathrm{H}_{2} \mathrm{O}(4.5 \mathrm{~mL})$. The resulting slurry was diluted with $\mathrm{Et}_{2} \mathrm{O}$, filtered through a pad of Celite $\left(\mathrm{Et}_{2} \mathrm{O}\right.$ rinse) and concentrated. Purification by dry-column flash chromatography ${ }^{34}$ (silica, $20-100 \%$ EtOAc/hexanes) provided D-rhamnal as a white solid (1.02 g, 59\% yield). $R_{\mathrm{f}} 0.37$ (EtOAc); mp $72-73^{\circ} \mathrm{C}$ (Et ${ }_{2} \mathrm{O} /$ hexanes) (lit. ${ }^{35} 71-73^{\circ} \mathrm{C}$ (EtOAc/hexanes)); ${ }^{1} \mathrm{H}$ NMR (500 MHz, $\left.\mathrm{CDCl}_{3}\right) \delta 6.32$ (dd, $J=$ 6.0, $1.6 \mathrm{~Hz}, 1 \mathrm{H}), 4.72(\mathrm{dd}, J=6.0,2.2 \mathrm{~Hz}, 1 \mathrm{H}), 4.21$ (app. d, $J=6.3 \mathrm{~Hz}, 1 \mathrm{H}), 3.87(\mathrm{dq}, J=9.8,6.3 \mathrm{~Hz}, 1 \mathrm{H})$, 3.43 (app. t, $J=8.6 \mathrm{~Hz}, 1 \mathrm{H}), 1.39$ (d, $J=6.3 \mathrm{~Hz}, 3 \mathrm{H})$.

\subsection{1,5-Anhydro-2,6-dideoxy-D-erythro-hex-1-enit-ulose 6}

To a stirred solution of D-rhamnal (100 mg, 0.768 mmol) in $\mathrm{CH}_{2} \mathrm{Cl}_{2}(7.5 \mathrm{~mL})$ at room temperature was added $\mathrm{MnO}_{2}(200 \mathrm{mg}, 2.30 \mathrm{mmol})$. After $3 \mathrm{~h}$, more $\mathrm{MnO}_{2}(200 \mathrm{mg}, 2.30 \mathrm{mmol})$ was added and the stirring was continued. After $15 \mathrm{~h}$, the reaction mixture was diluted with $\mathrm{CH}_{2} \mathrm{Cl}_{2}(10 \mathrm{~mL})$, filtered through a pad of Celite (EtOAc rinse) and concentrated. Purification by flash chromatography (silica, 40-50\% EtOAc/hexanes) provided $65 \mathrm{mg}$ of 6 as a white volatile solid $(66 \%$ yield). $R_{\mathrm{f}} 0.66$ (EtOAc); mp $91-92^{\circ} \mathrm{C}\left(\mathrm{Et}_{2} \mathrm{O} /\right.$ hexanes) (lit. ${ }^{36}$ 92-93 $\left.{ }^{\circ} \mathrm{C}\right) ;{ }^{1} \mathrm{H}$ NMR $\left(500 \mathrm{MHz}, \mathrm{CDCl}_{3}\right) \delta 7.39$ (app. d, $J=5.7 \mathrm{~Hz}, 1 \mathrm{H}), 5.46(\mathrm{~d}, J=5.7 \mathrm{~Hz}, 1 \mathrm{H}), 4.20$ $(\mathrm{dqd}, J=12.9,6.4,0.7 \mathrm{~Hz}, 1 \mathrm{H}), 3.54(\mathrm{~s}, 1 \mathrm{H}), 1.57(\mathrm{~d}$, $J=6.4 \mathrm{~Hz}, 3 \mathrm{H})$.

\subsection{1,5-Anhydro-2,6-dideoxy-4-O-nosyl-D-erythro-hex- 1-enit-3-ulose 7}

To a stirred solution of $6(0.212 \mathrm{~g}, 1.65 \mathrm{mmol})$ in $\mathrm{CH}_{2} \mathrm{Cl}_{2}(8.0 \mathrm{~mL})$ at $0^{\circ} \mathrm{C}$ were added pyridine $(0.667$ $\mathrm{mL}, 8.25 \mathrm{mmol})$ and nosyl chloride $(0.733 \mathrm{~g}, 3.31$ $\mathrm{mmol}$ ). The reaction mixture was allowed to warm to room temperature over $2 \mathrm{~h}$, and after stirring the mixture for a further $3 \mathrm{~h}$ more nosyl chloride $(0.367 \mathrm{~g}$, $1.66 \mathrm{mmol})$ and pyridine $(0.667 \mathrm{~mL}, 8.25 \mathrm{mmol})$ were added. After $12 \mathrm{~h}$ the reaction mixture was cooled to $0^{\circ} \mathrm{C}$ and water $(2 \mathrm{~mL})$ was added. After stirring for 30 min more water $(10 \mathrm{~mL})$ was added, the aqueous layer was separated and extracted with $\mathrm{CH}_{2} \mathrm{Cl}_{2}(2 \times 10 \mathrm{~mL})$, the combined organic layers were washed with brine (5 $\mathrm{mL})$, dried $\left(\mathrm{Na}_{2} \mathrm{SO}_{4}\right)$, filtered and concentrated. Purification by dry-columm flash chromatography (silica, $15-30 \%$ EtOAc/hexanes) provided 7 as a yellow crystalline solid $(0.411 \mathrm{~g}, 80 \%$ yield $) . R_{\mathrm{f}} 0.60(50 \%$ EtOAc/ hexanes); $\mathrm{mp} 93^{\circ} \mathrm{C} ;[\alpha]_{\mathrm{D}}+138.8\left(\mathrm{c} 1.07, \mathrm{CHCl}_{3}\right)$; IR (film) 3108, 1695, 1598, 1533, 1353, 1256, 1188, 1026, $854,780 \mathrm{~cm}^{-1} ;{ }^{1} \mathrm{H}$ NMR $\left(500 \mathrm{MHz}, \mathrm{CDCl}_{3}\right) \delta 8.39$ (app. d, $J=9.2 \mathrm{~Hz}, 2 \mathrm{H}$ ), 8.19 (app. d, $J=9.2 \mathrm{~Hz}, 2 \mathrm{H}$ ), $7.35(\mathrm{~d}, J=5.9 \mathrm{~Hz}, 1 \mathrm{H}), 5.37(\mathrm{~d}, J=5.9 \mathrm{~Hz}, 1 \mathrm{H}), 5.02$ $(\mathrm{d}, J=12.1 \mathrm{~Hz}, 1 \mathrm{H}), 4.54(\mathrm{dq}, J=12.1,6.2 \mathrm{~Hz}, 1 \mathrm{H})$, $1.63(\mathrm{~d}, J=6.2 \mathrm{~Hz}, 3 \mathrm{H}) ;{ }^{13} \mathrm{C}$ NMR $\left(125 \mathrm{MHz}, \mathrm{CDCl}_{3}\right)$ $\delta 180.0,163.5,150.8,142.1,129.7,124.1,105.0,79.6$, 77.4, 17.4; HRMS (MALDI) calcd for $\mathrm{C}_{12} \mathrm{H}_{11} \mathrm{NO}_{7} \mathrm{~S}$ $\left(\mathrm{M}^{+}\right) \mathrm{m} / z: 313.0256$, found 313.0286. 
4.4. 1,5-Anhydro-4-azido-2,6-dideoxy-D-threo-hex-1enit-3-ulose 8

To a stirred solution of $7(190 \mathrm{mg}, 0.606 \mathrm{mmol})$ in $\mathrm{CH}_{2} \mathrm{Cl}_{2}(3.3 \mathrm{~mL})$ at $0^{\circ} \mathrm{C}$ was added $n-\mathrm{Bu}_{4} \mathrm{NN}_{3}(0.651 \mathrm{~g}$, $2.29 \mathrm{mmol})$ [CAUTION] ${ }^{19}$ in $\mathrm{CH}_{2} \mathrm{Cl}_{2}(0.5 \mathrm{~mL})$ via cannula. The reaction mixture was allowed to warm to room temperature over $1.5 \mathrm{~h}$ and stirred at that temperature for $1 \mathrm{~h}$. Water $(5 \mathrm{~mL})$ was added, the aqueous layer was extracted with $\mathrm{CH}_{2} \mathrm{Cl}_{2}(2 \times 10 \mathrm{~mL})$, the combined organic extracts were washed with brine $(5 \mathrm{~mL})$ and dried $\left(\mathrm{Na}_{2} \mathrm{SO}_{4}\right)$, filtered and concentrated. Purification by dry-column flash chromatography (silica, 10$20 \%$ EtOAc/hexanes) provided 8 as a yellow oil $(88 \mathrm{mg}$, $95 \%$ yield). $R_{\mathrm{f}} 0.43\left(33 \%\right.$ EtOAc/hexanes); $[\alpha]_{\mathrm{D}}-78.0(c$ $0.383, \mathrm{CHCl}_{3}$ ); IR (film) 3378, 2919, 2106, 1675, 1593, $1414,1274,1050 \mathrm{~cm}^{-1} ;{ }^{1} \mathrm{H}$ NMR $\left(500 \mathrm{MHz}, \mathrm{CDCl}_{3}\right) \delta$ $7.37(\mathrm{~d}, J=6.0 \mathrm{~Hz}, 1 \mathrm{H}), 5.49(\mathrm{dd}, J=6.0,0.9 \mathrm{~Hz}, 1 \mathrm{H})$, $4.53(\mathrm{qd}, J=6.6,3.1 \mathrm{~Hz}, 1 \mathrm{H}), 3.81(\mathrm{~d}, J=3.1 \mathrm{~Hz}, 1 \mathrm{H})$, $1.48(\mathrm{~d}, J=6.6 \mathrm{~Hz}, 3 \mathrm{H}) ;{ }^{13} \mathrm{C}$ NMR $\left(100 \mathrm{MHz}, \mathrm{CDCl}_{3}\right)$ $\delta$ 187.1, 163.6, 104.9, 76.9, 63.8, 15.3; HRMS (MALDI) calcd for $\mathrm{C}_{6} \mathrm{H}_{8} \mathrm{NO}_{2}\left(\mathrm{MH}-\mathrm{N}_{2}{ }^{+}\right) \mathrm{m} / z: 126.0550$, found 126.0554 .

\subsection{1,5-Anhydro-4-azido-2,6-dideoxy-3- $C$-methyl-D- lyxo-hex-1-enitol 9}

To a stirred solution of $8(95 \mathrm{mg}, 0.620 \mathrm{mmol})$ in THF $(3.1 \mathrm{~mL})$ at $-100^{\circ} \mathrm{C}$ was added $\mathrm{MeLi}(1.5 \mathrm{M}$ solution in $\mathrm{Et}_{2} \mathrm{O}, 0.500 \mathrm{~mL}, 0.744 \mathrm{mmol}$ ) dropwise. After $1.5 \mathrm{~h}$, sat. aq. $\mathrm{NH}_{4} \mathrm{Cl}(1.0 \mathrm{~mL})$ was added and the reaction mixture was allowed to warm to room temperature. The aqueous layer was extracted with EtOAc $(2 \times 5 \mathrm{~mL})$, the combined organic extracts were washed with brine (5 $\mathrm{mL})$, dried $\left(\mathrm{Na}_{2} \mathrm{SO}_{4}\right)$, filtered and concentrated. Purification by dry-column flash chromatography (silica, 10 $25 \%$ EtOAc/hexanes) provided 9 as a yellow oil $(81 \mathrm{mg}$, $77 \%$ yield). $R_{\mathrm{f}} 0.15(20 \%$ EtOAc/hexanes $) ;[\alpha]_{\mathrm{D}}+84.2(c$ $0.483, \mathrm{CHCl}_{3}$ ); IR (film) 3413, 2919, 2097, 1637, 1449, $1378,1232,1132,1049,944,820,750 \mathrm{~cm}^{-1} ;{ }^{1} \mathrm{H}$ NMR $\left(500 \mathrm{MHz}, \mathrm{CDCl}_{3}\right) \delta 6.27(\mathrm{~d}, J=6.3 \mathrm{~Hz}, 1 \mathrm{H}), 4.70(\mathrm{dd}$, $J=6.3,2.2 \mathrm{~Hz}, 1 \mathrm{H}$ ), 4.19 (qd, $J=6.5,0.9 \mathrm{~Hz}, 1 \mathrm{H}$ ), 3.34 (br s, 1H), 2.24 (br s, 1H) 1.45 (d, $J=6.6 \mathrm{~Hz}, 3 \mathrm{H}), 1.43$ (s, 3H); ${ }^{13} \mathrm{C}$ NMR $\left(100 \mathrm{MHz}, \mathrm{CDCl}_{3}\right) \delta 143.6,104.8$, 72.1, 68.8, 68.4, 29.5, 18.0. Low resolution mass spectrum $(\mathrm{GC} / \mathrm{MS})$ calcd for $\mathrm{C}_{7} \mathrm{H}_{11} \mathrm{~N}_{3} \mathrm{O}_{2}\left(\mathrm{M}^{+}\right) 169$, found 169.

\subsection{Methyl 4-azido-4,6-dideoxy-3- $C$-methyl- $\alpha$-D-talo- pyranoside 10}

To a stirred solution of $9(100 \mathrm{mg}, 0.591 \mathrm{mmol}, 100$ $\mathrm{mol} \%)$ in $\mathrm{MeOH}(3.1 \mathrm{~mL})$ at $0^{\circ} \mathrm{C}$ were added $\mathrm{NaHCO}_{3}$ (153 $\mathrm{mg}, 1.86 \mathrm{mmol}, 315 \mathrm{~mol} \%$ ) and $m$-CBPA (55-60\%, $0.321 \mathrm{~g}, 1.86 \mathrm{mmol}, 315 \mathrm{~mol} \%)$. After stirring for $1 \mathrm{~h}$ sat. aq. $\mathrm{NaHCO}_{3}(2 \mathrm{~mL})$ was added, the aqueous layer was extracted with EtOAc $(2 \times 10 \mathrm{~mL})$, the combined organic extracts were washed with brine $(5 \mathrm{~mL})$ and dried $\left(\mathrm{Na}_{2} \mathrm{SO}_{4}\right)$, filtered and concentrated. Purification by flash chromatography (silica, 20-30\% EtOAc/hexanes) provided 10 as an oil (76 $\mathrm{mg}, 60 \%$ yield). $R_{\mathrm{f}} 0.28$ $\left(33 \%\right.$ EtOAc/hexanes); $[\alpha]_{\mathrm{D}}+143.8\left(c 0.417, \mathrm{CHCl}_{3}\right) ; \mathrm{IR}$ (film) 3449, 2921, 2109, 1449, 1343, 1261, 1126, 1061,
997, 944, $814 \mathrm{~cm}^{-1} ;{ }^{1} \mathrm{H}$ NMR (500 MHz, $\left.\mathrm{CDCl}_{3}\right) \delta 4.76$ $(\mathrm{s}, 1 \mathrm{H}), 4.03(\mathrm{qd}, J=6.5,1.2 \mathrm{~Hz}, 1 \mathrm{H}), 3.62(\mathrm{br} \mathrm{s}, 1 \mathrm{H})$, $3.36(\mathrm{~s}, 3 \mathrm{H}), 3.30(\mathrm{~d}, J=11.0 \mathrm{~Hz}, 1 \mathrm{H}), 3.24(\mathrm{~s}, 1 \mathrm{H}), 2.71$ $(\mathrm{d}, J=11.0 \mathrm{~Hz}, 1 \mathrm{H}), 1.39(\mathrm{~s}, 3 \mathrm{H}), 1.34(\mathrm{~d}, J=6.5 \mathrm{~Hz}$, $3 \mathrm{H}) ;{ }^{13} \mathrm{C}$ NMR $\left(100 \mathrm{MHz}, \mathrm{CDCl}_{3}\right) \delta 102.0,73.2,70.0$, 64.2, 55.5, 23.5, 18.1; HRMS (MALDI) calcd for $\mathrm{C}_{8} \mathrm{H}_{15} \mathrm{~N}_{3} \mathrm{O}_{4} \mathrm{Na} \quad\left(\mathrm{M}+\mathrm{Na}^{+}\right) \quad m / z: \quad 240.0955$, found 240.0956 .

\subsection{Methyl 4-azido-4,6-dideoxy-3-C,2-O-dimethyl- $\alpha$-D- talo-pyranoside}

To a stirred solution of $\mathbf{1 0}$ (70 $\mathrm{mg}, 0.322 \mathrm{mmol})$ in $\mathrm{Et}_{2} \mathrm{O}$ $(2.0 \mathrm{~mL})$ at room temperature were added MeI $(0.200$ $\mathrm{mL}, 3.22 \mathrm{mmol})$ and freshly prepared $\mathrm{Ag}_{2} \mathrm{O}^{29}(0.224 \mathrm{~g}$, $0.967 \mathrm{mmol})$. The reaction mixture was protected from light and heated to reflux. After stirring under reflux for $5 \mathrm{~h}$, the reaction was diluted with EtOAc $(10 \mathrm{~mL})$, filtered through a pad of Celite (EtOAc rinse) and concentrated. Purification by flash chromatography (silica, 5-15\% EtOAc/hexanes) provided the azidomethyl glycoside as an oil (53 $\mathrm{mg}, 72 \%$ yield). $R_{\mathrm{f}} 0.33(33 \%$ EtOAc/hexanes); $[\alpha]_{\mathrm{D}}+164.8\left(c \quad 0.708, \mathrm{CHCl}_{3}\right) ;$ IR (film) $3483,2931,2105,1455,1349,1102,1055,597$ $\mathrm{cm}^{-1} ;{ }^{1} \mathrm{H}$ NMR $\left(400 \mathrm{MHz}, \mathrm{CDCl}_{3}\right) \delta 4.79(\mathrm{~s}, 1 \mathrm{H}), 4.00$ $(\mathrm{qd}, J=6.5,1.6 \mathrm{~Hz}, 1 \mathrm{H}), 3.72(\mathrm{~d}, J=1.0 \mathrm{~Hz}, 1 \mathrm{H}), 3.49$ (s, 3H), $3.36(\mathrm{~s}, 3 \mathrm{H}), 3.11$ (br s, 1H) $2.90(\mathrm{~s}, 1 \mathrm{H}), 1.40$ $(\mathrm{d}, J=1.0 \mathrm{~Hz}, 3 \mathrm{H}), 1.35(\mathrm{~d}, J=6.5 \mathrm{~Hz}, 3 \mathrm{H}) ;{ }^{13} \mathrm{C} \mathrm{NMR}$ $\left(125 \mathrm{MHz}, \mathrm{CDCl}_{3}\right) \delta 98.4,82.0,70.1,69.6,64.3,59.8$, 55.2, 24.5, 17.9; HRMS (MALDI) calcd for $\mathrm{C}_{9} \mathrm{H}_{17} \mathrm{~N}_{3} \mathrm{O}_{4} \mathrm{Na} \quad\left(\mathrm{M}+\mathrm{Na}^{+}\right) \quad m / z:$ 254.1111, found 254.1112 .

\subsection{Methyl 4-(tert-butoxycarbonylamino)-4,6-dideoxy- 3-C,2-O-dimethyl- $\alpha$-D-talo-pyranoside 11}

To a stirred solution of the methyl glycoside $(26 \mathrm{mg}$, $0.112 \mathrm{mmol})$ and $\mathrm{Boc}_{2} \mathrm{O}(49 \mathrm{mg}, 0.225 \mathrm{mmol})$ in EtOAc $(0.50 \mathrm{~mL})$ at room temperature under argon was carefully added $\mathrm{Pd} / \mathrm{C}(10 \%, 2 \mathrm{mg})$. The reaction flask was evacuated and placed under an $\mathrm{H}_{2}$ atmosphere. After 4 $\mathrm{h}$ more $\mathrm{Pd} / \mathrm{C}(10 \%, 2 \mathrm{mg})$ was added. After stirring for $1 \mathrm{~h}$ the reaction flask was purged with argon, the mixture was filtered through a pad of Celite and concentrated. Purification by flash chromatography (silica, $10-20 \% \mathrm{EtOAc} /$ hexanes with $2 \% \mathrm{Et}_{3} \mathrm{~N}$ ) provided 11 as an oil $\left(21 \mathrm{mg}, 62 \%\right.$ yield). $R_{\mathrm{f}} 0.28(33 \% \mathrm{EtOAc} / \mathrm{hex}-$ anes); $[\alpha]_{\mathrm{D}}+59.4\left(c 0.500, \mathrm{CHCl}_{3}\right)$ IR (film) 3429, 2929, 1716, 1503, 1170, $1060 \mathrm{~cm}^{-1}$; ${ }^{1} \mathrm{H}$ NMR $(500 \mathrm{MHz}$, $\left.\mathrm{CDCl}_{3}\right) \delta 5.30(\mathrm{~d}, J=10.4 \mathrm{~Hz}, 1 \mathrm{H}), 4.73(\mathrm{~s}, 1 \mathrm{H}), 4.01$ $(\mathrm{qd}, J=6.4,1.7 \mathrm{~Hz}, 1 \mathrm{H}), 3.49(\mathrm{dd}, J=10.4,1.7 \mathrm{~Hz}$, $1 \mathrm{H}), 3.47(\mathrm{~s}, 3 \mathrm{H}), 3.37(\mathrm{~s}, 3 \mathrm{H}), 3.23(\mathrm{~s}, 1 \mathrm{H}) 2.91(\mathrm{~s}, 1 \mathrm{H})$, $1.44(\mathrm{~s}, 9 \mathrm{H}), 1.41(\mathrm{~s}, 3 \mathrm{H}), 1.15(\mathrm{~d}, J=6.4 \mathrm{~Hz}, 3 \mathrm{H}) ;{ }^{13} \mathrm{C}$ NMR $\left(150 \mathrm{MHz}, \mathrm{CDCl}_{3}\right) \delta 156.5,98.6,83.0,79.3,68.0$, 65.0, 59.4, 58.4, 55.2, 28.3, 23.8, 17.1; HRMS (MALDI) calcd for $\mathrm{C}_{14} \mathrm{H}_{27} \mathrm{NO}_{6} \mathrm{Na}\left(\mathrm{M}+\mathrm{Na}^{+}\right) m / z: 328.1730$, found 328.1731 .

\section{9. $\alpha$-D-Methyl callipeltose 12}

To a stirred solution of $11(28 \mathrm{mg}, 0.0917 \mathrm{mmol})$ in THF $(0.50 \mathrm{~mL})$ at $0^{\circ} \mathrm{C}$ was added $t$-BuOK $(0.9 \mathrm{M}$ solution in THF, $0.204 \mathrm{~mL}, 0.183 \mathrm{mmol}$ ) dropwise and 
the cooling bath was removed. After $4 \mathrm{~h}$ at room temperature more $t$-BuOK $(0.051 \mathrm{~mL}, 0.46 \mathrm{mmol})$ was added. After $1 \mathrm{~h}$ sat. aq. $\mathrm{NH}_{4} \mathrm{Cl}(1 \mathrm{~mL})$ was added, the aqueous layer was extracted with EtOAc $(2 \times 5 \mathrm{~mL})$, the combined organic extracts were washed with brine (5 $\mathrm{mL})$ and dried $\left(\mathrm{Na}_{2} \mathrm{SO}_{4}\right)$, filtered and concentrated. Purification by flash chromatography (silica, 1-3\% $\mathrm{MeOH} / \mathrm{CH}_{2} \mathrm{Cl}_{2}$ ) provided $15 \mathrm{mg}$ of $\mathbf{1 2}$ as a white solid $\left(69 \%\right.$ yield). $R_{\mathrm{f}} 0.28\left(5 \% \mathrm{MeOH} / \mathrm{CH}_{2} \mathrm{Cl}_{2}\right) ; \mathrm{mp} 145-$ $146^{\circ} \mathrm{C}$ (hexanes) (lit. $\left.{ }^{8} 147-148^{\circ} \mathrm{C}\right) ;[\alpha]_{\mathrm{D}}+91.6(c$ 1.06, $\left.\mathrm{CHCl}_{3}\right)\left(\right.$ lit. $\left.^{9}[\alpha]_{\mathrm{D}}+76\left(c=1.0, \mathrm{CHCl}_{3}\right)\right)$; IR (film) 3295, 2931, 1747, 1376, 1267, 1104, 1060, $670 \mathrm{~cm}^{-1}$; ${ }^{1} \mathrm{H}$ NMR $\left(500 \mathrm{MHz}, \mathrm{MeOH}-d_{4}\right) \delta 4.48(\mathrm{~d}, J=6.1 \mathrm{~Hz}, 1 \mathrm{H}), 3.94$ $(\mathrm{qd}, J=6.5,2.0 \mathrm{~Hz}, 1 \mathrm{H}), 3.52(\mathrm{~s}, 3 \mathrm{H}), 3.45(\mathrm{~d}, J=2.0$ $\mathrm{Hz}, 1 \mathrm{H}), 3.41(\mathrm{~s}, 3 \mathrm{H}), 3.38(\mathrm{~d}, J=6.1 \mathrm{~Hz}, 1 \mathrm{H}), 1.50(\mathrm{~s}$, $3 \mathrm{H}), 1.12(\mathrm{~d}, J=6.5 \mathrm{~Hz}, 3 \mathrm{H}) ;{ }^{1} \mathrm{H}$ NMR $(400 \mathrm{MHz}$, $\left.\mathrm{CDCl}_{3}\right) \delta 6.69(\mathrm{br} \mathrm{s}, 1 \mathrm{H}), 4.60(\mathrm{~d}, J=5.6 \mathrm{~Hz}, 1 \mathrm{H}), 3.89$ (qd, $J=6.5,2.0 \mathrm{~Hz}, 1 \mathrm{H}), 3.53(\mathrm{~s}, 3 \mathrm{H}), 3.41(\mathrm{~s}, 3 \mathrm{H}), 3.37$ $(\mathrm{d}, J=2.0 \mathrm{~Hz}, 1 \mathrm{H}), 3.19(\mathrm{~d}, J=5.6 \mathrm{~Hz}, 1 \mathrm{H}), 1.54(\mathrm{~s}$, $3 \mathrm{H}), 1.17(\mathrm{~d}, J=6.5 \mathrm{~Hz}, 3 \mathrm{H}) ;{ }^{13} \mathrm{C}$ NMR $(100 \mathrm{MHz}$, $\left.\mathrm{CDCl}_{3}\right) \delta 159.1,101.6,81.9,81.1,63.7,61.4,60.6,55.0$, 23.3, 15.6; HRMS (MALDI) calcd for $\mathrm{C}_{10} \mathrm{H}_{17} \mathrm{NO}_{5} \mathrm{Na}$ $\left(\mathrm{M}+\mathrm{Na}^{+}\right) \mathrm{m} / z: 254.0999$, found 254.1001.

\subsection{0. $\alpha$-D-Callipeltose 4}

A solution of $12(4.3 \mathrm{mg}, 0.186 \mathrm{mmol})$ in $\mathrm{H}_{2} \mathrm{SO}_{4}(2 \mathrm{M}$ in $\mathrm{H}_{2} \mathrm{O} / 1$,4-dioxane $\left.(1: 1), 0.20 \mathrm{~mL}\right)$ was heated to $60^{\circ} \mathrm{C}$ for $30 \mathrm{~h}$. Saturated aq. $\mathrm{NaHCO}_{3}(1 \mathrm{~mL})$ was added, the solution was saturated with $\mathrm{NaCl}$ and extracted with $25 \% \mathrm{EtOH} / \mathrm{CHCl}_{3}(20 \times 1 \mathrm{~mL})$. The combined organic extracts were washed with brine and dried $\left(\mathrm{Na}_{2} \mathrm{SO}_{4}\right)$, filtered and concentrated. Purification by flash chromatography (silica, $5 \% \mathrm{MeOH} / \mathrm{CH}_{2} \mathrm{Cl}_{2}$ ) afforded D-callipeltose 4 as a film (1.9 mg, 48\% yield). $R_{\mathrm{f}} 0.13(5 \%$ $\mathrm{MeOH} / \mathrm{CH}_{2} \mathrm{Cl}_{2}$ ); $[\alpha]_{\mathrm{D}}+25.3$ ( c $0.150, \mathrm{CHCl}_{3}$ ); IR (film) $3313,2924,1737,1449,1273,1261,1061,667 \mathrm{~cm}^{-1} ;{ }^{1} \mathrm{H}$ NMR $\left(500 \mathrm{MHz}, \mathrm{CDCl}_{3}\right) \delta 5.56(\mathrm{~s}, 1 \mathrm{H}), 5.13(\mathrm{~d}, J=5.9$ $\mathrm{Hz}, 1 \mathrm{H}), 4.06(\mathrm{qd}, J=6.5,1.9 \mathrm{~Hz}, 1 \mathrm{H}), 3.60(\mathrm{~s}, 3 \mathrm{H})$, $3.38(\mathrm{~d}, J=1.9 \mathrm{~Hz}, 1 \mathrm{H}), 3.24(\mathrm{~d}, J=5.9 \mathrm{~Hz}, 1 \mathrm{H}), 2.99$ (br s, $1 \mathrm{H}), 1.56(\mathrm{~s}, 3 \mathrm{H}), 1.17(\mathrm{~d}, J=6.5 \mathrm{~Hz}, 3 \mathrm{H}) ;{ }^{13} \mathrm{C}$ NMR $\left(150 \mathrm{MHz}, \mathrm{CDCl}_{3}\right) \delta 158.3,95.0,81.9,81.8,63.6$, $61.3,60.8,23.3,15.8$; HRMS (MALDI) calcd for $\mathrm{C}_{9} \mathrm{H}_{15} \mathrm{NO}_{5} \mathrm{Na}\left(\mathrm{M}+\mathrm{Na}^{+}\right) \mathrm{m} / z: 240.0842$, found 240.0843 .

\section{Acknowledgements}

We thank Dr. D. H. Huang and Dr. G. Siuzdak for NMR spectroscopic and mass spectrometric assistance, respectively. Financial support for this work was provided by The Skaggs Institute for Chemical Biology, The Ministry of Education Graduate School Program (Finland) and the Emil Aaltonen Foundation (both to A.J.P.).

\section{References}

1. Zampella, A.; D'Auria, M. V.; Minale, L.; Debitus, C.; Roussakis, C. J. Am. Chem. Soc. 1996, 118, 11085-11088.
2. Zampella, A.; D’Auria, M. V.; Minale, L.; Debitus, C. Tetrahedron 1997, 53, 3243-3248.

3. Hoye, T. R.; Zhao, H. Org. Lett. 1999, 1, 169-177.

4. Velázquez, F.; Olivo, H. F. Org. Lett. 2000, 2, 1931-1933.

5. Olivo, H. F.; Velázquez, F.; Trevisan, H. C. Org. Lett. 2000, 2, 4055-4058.

6. Paterson, I.; Davies, R. D. M.; Marquez, R. Angew. Chem., Int. Ed. 2001, 40, 603-607.

7. Evans, D. A.; Burch, J. D. Org. Lett. 2001, 2, 503-505.

8. Smith, G. R.; Finley, IV, J. J.; Giuliani, R. M. Carbohydr. Res. 1998, 308, 223-227.

9. Gurjar, M. K.; Reddy, R. Carbohydr. Lett. 1998, 3, 169-172.

10. On a larger scale, D-glucal can be prepared from tri- $O$ acetyl-D-glucal by saponification of the three acetyl groups with $\mathrm{K}_{2} \mathrm{CO}_{3}$ in $\mathrm{MeOH}$.

11. Brimacombe, J. S.; Da'aboul, I.; Tucker, L. C. N. Carbohydr. Res. 1971, 19, 276-280.

12. Boivin, J.; Montagnac, A.; Monneret, C.; Païs, M. Carbohydr. Res. 1980, 85, 223-242.

13. Fraser-Reid, B.; Kelly, D. R.; Tulshian, D. B.; Ravi, P. S. J. Carbohydr. Chem. 1983, 2, 105-114.

14. Thiem, J.; Elvers, J. Chem. Ber. 1981, 114, 1442-1454.

15. Csuk, R.; Huegener, M.; Vasella, A. Helv. Chim. Acta 1988, 71, 609-618.

16. Hanessian, S.; Vatèle, J.-M. Tetrahedron Lett. 1981, 22, 3579-3582.

17. Nicolaou, K. C.; Li, J.; Zenke, G. Helv. Chim. Acta 2000, 83, 1977-2006.

18. Preparation of tetra- $n$-butylammonium azide: Brändström, A.; Lamm, B.; Palmertz, I. Acta Chem. Scand. B 1974, 28, 699-701. Commercially available from TCI America. The commercial material was used in this synthesis.

19. Solutions of tetra- $n$-butylammonium azide in $\mathrm{CH}_{2} \mathrm{Cl}_{2}$ are reported to form explosive products on storage. If stored for longer times, the compound should be dissolved in toluene, which gives a stable solution: Hansson, T. G.; Kihlberg, J. O. J. Org. Chem. 1986, 51, 4490-4492.

20. $\beta$-Keto azides have been found to be prone to epimerization: Evans, D. A.; Britton, T. C.; Ellman, J. A.; Dorow, R. L. J. Am. Chem. Soc. 1990, 112, 4011-4030.

21. The C-(4) epimer was also removed at this stage.

22. Nicolaou, K. C.; Groneberg, R. D. J. Am. Chem. Soc. 1990, 112, 4085-4086.

23. Barili, P. L.; Berti, G.; Catelani, G.; D’Andrea, F. Tetrahedron Lett. 1991, 32, 959-962.

24. Bellucci, G.; Catelani, G.; Chiappe, C.; D’Andrea, F. Tetrahedron Lett. 1994, 35, 8433-8436.

25. Upreti, M.; Vishwakarma, R. A. Tetrahedron Lett. 1999, 40, 2619-2622.

26. Saleh, T.; Rousseau, G. Synlett 1999, 617-619.

27. Rainier, J. D.; Allwein, S. P.; Cox, J. M. Org. Lett. 2000 , 2, 231-234.

28. Rainier, J. D.; Allwein, S. P.; Cox, J. M. J. Org. Chem. 2001, 66, 1380-1386.

29. Silver(I) oxide was prepared according to: Pearl, I. A. Org. Syn. Coll. Vol. IV, 972-973, washed with $\mathrm{MeOH}$ and $\mathrm{Et}_{2} \mathrm{O}$ and dried in vacuo.

30. Saito, S.; Nakajima, H.; Inaba, M.; Moriwake, T. Tetrahedron Lett. 1989, 30, 837-838.

31. Bull, S. D.; Davies, S. G.; Jones, S.; Sanganee, H. J. J. 
Chem. Soc., Perkin Trans. 1 1999, 387-389. Giuliani et al. exploited a similar cyclization for the corresponding Cbzprotected amide using $\mathrm{NaH}{ }^{8}$

32. Brimacombe, J. S.; Rahman, K. M. M. J. Chem. Soc., Perkin Trans. 1 1985, 1067-1072.

33. Jütten, P.; Scharf, H.-D. Carbohydr. Res. 1991, 212,
93-108.

34. Harwood, L. M. Aldrichim. Acta 1985, 18, 25.

35. Horton, D.; Issa, M.; Priebe, W.; Szhaidman, M. L. Carbohydr. Res. 1993, 246, 105-118.

36. Czernecki, S.; Vijayakumaran, K.; Ville, G. J. Org. Chem. 1986, 51, 5472-5475. 